\title{
Research on Image Dimension Reduction Method Based on Non-negative Matrix Factorization and Discrete Cosine Transform
}

\author{
Panlong Sun ${ }^{1, a}$ and Qinghua Wang ${ }^{1, b}$ \\ ${ }^{1}$ School of Mechatronic Engineering, Xi'an Technological University, Xi'an, China, 710032 \\ aspl0305154549@163.com, b122133416@qq.com
}

Keywords: NMF; DCT; Image dimension reduction; Compression

\begin{abstract}
According to the denoising technique based on the non-negative matrix factorization (NMF) and Redundancy technique based on discrete cosine transform (DCT), a fusion method is proposed, namely, NMF-DCT dimension reduction method. This method is applied to the dimension reduction of ORL face images. The image reconstruction degree and compression ratio are used to be as evaluation criterion. The experimental results show that the NMF-DCT method is more effective than only use the NMF or only use the DCT. The NMF-DCT method has a good effect on the face image compression.
\end{abstract}

\section{Introduction}

Image is an important source for human to obtain information. Today, with the rapid development of information technology and photographic pixels, which brought us inconvenient to use and transfer images. Therefore, an efficient dimension reduction method needs to be used to reduce the dimension of the image [1]. The traditional dimension method is divided into linear and non-linear. The NMF method is one of non-linear dimension reduction methods. It was proposed by D. D. Lee and H. S. Seung in 1999 [2]. In the NMF method, the original matrix is expressed as the product of the coefficient matrix and the matrix. And the resulting two decomposition matrixes are non-negative. Based on these characteristics, the computation of the data processing is greatly reduced [3].

The NMF method can only reduce the dimension but cannot remove the redundancy. In order to obtain the optimal dimension, it is necessary to compress the images. The problem to be solved is to minimize the amount of data to represent a digital image [4]. Therefore, the NMF method is combined with the DCT method to achieve the purpose of reducing dimension and remove the redundancy, which is called NMF-DCT method.

\section{NMF-DCT Method}

NMF Implementation Principle. In fact, the NMF solution problem is an optimal solution problem, and the method of multiplicative iteration is used to solve the $W$ and $H$. There are many kinds of objective functions for NMF problem, and the most widely used are Euclidean distance and $K L$ divergence.

In the decomposition of NMF, we assume that the noise matrix is $E \in R^{n \times m}$, so

$E=V-W H$

Now find the right one $W$ and $H$ make $\|E\|$ the smallest.

You can then iterate using the steepest descent method. as follows

$$
\begin{aligned}
& W_{i k}=W_{i k}-\partial_{1} \cdot\left\lfloor\left(V H^{T}\right)_{i k}-\left(W H H^{T}\right)_{i k}\right\rfloor \\
& H_{k j}=H_{k j}-\partial_{2} \cdot\left\lfloor\left(W^{T} V\right)_{k i}-\left(W^{T} W H\right)_{k j}\right\rfloor
\end{aligned}
$$

Select 


$$
\begin{aligned}
& \partial_{1}=\frac{W_{i k}}{\left(W^{T} W H\right)_{i k}} \\
& \partial_{2}=\frac{H_{k j}}{\left(W H H^{T}\right)_{k j}}
\end{aligned}
$$

Then the final iteration is

$$
\begin{aligned}
& W_{i k}=W_{i k} \cdot \frac{\left(V H^{T}\right)_{i k}}{\left(W H H^{T}\right)_{i k}} \\
& H_{k j}=H_{k j} \cdot \frac{\left(W^{T} V\right)_{k j}}{\left(W^{T} W H\right)_{k j}}
\end{aligned}
$$

By Eq.6, Eq.7 can be seen that this is multiplicative iterative rules, each step to ensure that the results are positive, iteration continue will converge [5,6]. Let $v_{i}$ and $h_{i}$ denote the column $\mathrm{i}$ vector of $V$ and $H$, respectively, then $V \approx W H$ can be transformed into the form of expression using column vectors: $v_{i} \approx W h_{i}$. That is each $v_{i}$ can roughly be a linear combinations of column vectors of the base matrix $W$, the weight of the combination is a component of $h_{i}$. Thus, non-negative matrix $W$ can be regarded as a set of bases for projection of the original matrix $V$, and the matrix $H$ can be regarded as the mapping of the original matrix $V$ in the vector space $W$.

DCT Transform Principle. Assuming that the image matrix is $V=f(x, y)$, which is a matrix $m \times n$, the two-dimensional discrete Fourier transform of the matrix is defined as follows:

$$
\begin{aligned}
& F(0,0)=\frac{1}{\sqrt{m n}} \sum_{x=0}^{m-1} \sum_{y=0}^{n-1} f(x, y) \\
& F(u, v)=\frac{2}{\sqrt{m n}} \sum_{x=0}^{m-1} \sum_{y=0}^{n-1} f(x, y)\left[\cos \frac{(2 x+1) u \pi}{2 m}\right]\left[\cos \frac{(2 y+1) v \pi}{2 n}\right]
\end{aligned}
$$

among them $x, v=0,1, \ldots m-1 ; v=0,1, \ldots, n-1$.

After DCT, the low frequency signal is concentrated in the upper left corner of the image, and the high frequency signal is concentrated in the lower right corner of the image. This is determined by the discrete cosine transform 'energy concentration [7].

NMF-DCT Principle. NMF-DCT principle is based on the principle of non-negative matrix decomposition and discrete cosine transform. Firstly, the original matrix $V$ is dimension reduced by NMF, and the reduced base matrix $W$ and the projection coefficient matrix $m y H$ are obtained. Then the NMF multiplication principle is used to multiply the two matrices to obtain the reconstructed matrix $V^{\prime}$. Then, the reconstructed matrix is $V^{\prime}$ compressed by DCT, so the dimension reduction is achieved.

\section{Dimension Reduction Dimension of Image}

Fit Degree. Used $P_{i}$ to represent the degree of agreement between two matrices, can be used to measure the degree of similarity matrix. Therefore, when the original matrix and the reduced dimension compressed matrix are known, the dimension reduction effect of the different dimension reduction methods can be compared by calculating the fit degree between the original matrix and the compression matrix [8].

The fit degree between the matrix $V_{i}$ and $V_{i}^{\prime}$ is defined as: 


$$
P_{i}=\left(\frac{\left\|V_{i}-V_{i}^{\prime}\right\|^{2}}{\left\|V_{i}\right\|^{2}}\right) \times 100 \%
$$

$V_{i}$ represents the original image matrix, and $V_{i}^{\prime}$ is the image matrix after the reduced dimension reconstruction. When $P_{i}=100 \%$ the matrix after dimension reduction is consistent with the original matrix. The matrix and the projection coefficient matrix are reconstructed by MATLAB, and the reconstructed degrees are compared.

Compression Ratio. The introduction of distortion is unavoidable when compressing the image. In the user can not perceive and can tolerate the premise of distortion, in exchange for higher coding efficiency we want to further improve the compression ratio [9]. Here we introduce the compression ratio of this indicator: $\mathrm{Cr}=\frac{n_{1}}{n_{2}}\left(n_{1}\right.$ is the original image of the number of bits, $n_{2}$ is the number of bits after compression).

In this paper, the reconstructed image with the dimension $r$ of 50 is compressed to find the ratio of the original image to the compressed image. And compared with the NMF and DCT methods alone.

\section{Non-Negative Matrix Factorization and Discrete Cosine Transform are Applied to Image Dimension Reduction}

This paper selects one face image from the ORL face database to do the experiment, and expresses the face image with $v$. The size of the face image is $112 \times 92$, where $\mathrm{m}$ is the row number of the matrix and $\mathrm{n}$ is the number of the columns. Using NMF to reduce the dimension of the data, $r$ is reduced by 20,30, 40, 50 and 60 respectively. In this experiment, the number of times of iteration termination is kept constant at 200. Theoretically, the more iterations, the closer to the original values. Reach the base matrix $W$ and the projection coefficient matrix $m y H$, then the matrix $V^{\prime}$ is reconstructed by the NMF multiplicative principle. This experiment is programmed in MATLAB. Specific experimental steps are as follows:

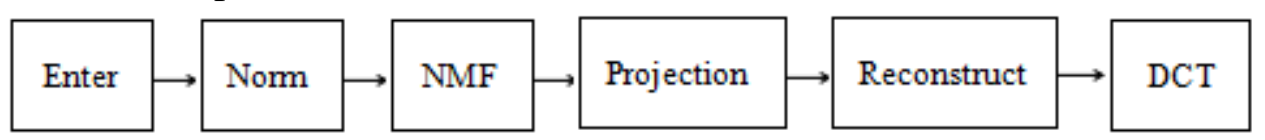

Figure 1. Experimental flow chart

Step 1 Use the imread function in MATLAB to read the first image of the first person in orl-faces as the experimental sample to generate the original matrix $V$. The generated matrix $V$ is a $112 \times$ 92 matrix.

Step 2 The original matrix data $V$ is normalized, and the sum of the normalized rear column vectors is 1. A normalized matrix $n v$ is obtained.

Step 3 Using MATLAB to write the non-negative matrix factorization program to get the reduced base matrix $W$ and coefficient matrix $H$.

Step 4 The normalized matrix $n v$ is projected onto the base matrix $W$ generated in the previous step to obtain the new coefficient matrix $m y H$.

Step 5 Using the multiplication principle of non-negative matrix factorization, the matrix $W$ obtained in step 4 and the coefficient matrix $m y H$ obtained in step 5 are multiplied to obtain the reconstructed matrix $V^{\prime}[10]$.

Here we observe the face image in the $r$ value of different values of the reconstruction effect. 


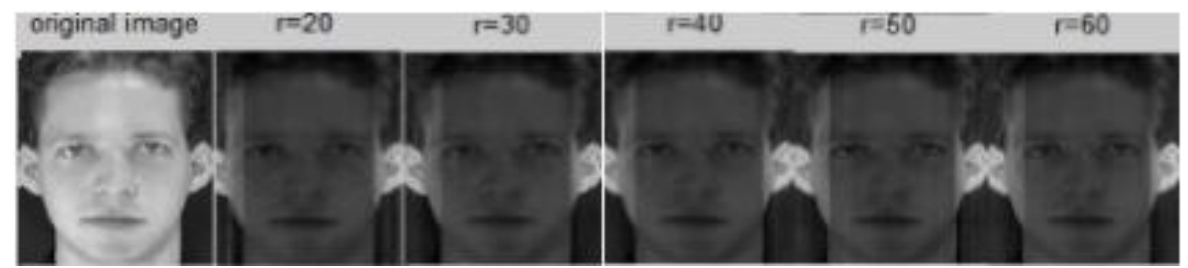

Figure 2. The original image and take different values $r$ of the reconstruction image

The following table is $r$ taken from different values, the reconstructed image coincides with the original image.

Table 1 Reconstructed image fit degree

\begin{tabular}{cccccc}
\hline R value & 20 & 30 & 40 & 50 & 60 \\
NMF fit degree $\%$ & 96.21 & 98.37 & 98.83 & 99.64 & 99.64 \\
\hline
\end{tabular}

From the above comparison can see the $r$ value of the reconstructed image of the match degree also has a great influence, from the results we can see that the best $r$ value in this experiment should be between 50-60, if the value of small, will lead to reconstruction of the low degree of consistency will make some useful information is lost, if the value is too large, you will lose the original data for the meaning of the dimension reduction. Obviously, select the 50 most appropriate here.

Step 6 Call MATLAB built-in detmtx function to reconstruct matrix $V^{\prime}$ when $r$ is 50 to DCT to get the compressed image. The transformation from the DCT transform will decompose the matrix into $8 \times 8$ sub-blocks for DCT transformation. Since the reconstructed matrix $V^{\prime}$ is a $112 \times$ 92 matrix, the program will automatically complete the compression Into the $N \times N$ type matrix, that is $112 \times 112$. The image matrix can be divided into 196 blocks.

The following figure is the use of DCT transform after compression of the face image before and after the contrast effect: (a .50-dimensional reconstruction of the image, b. For the DCT compressed image)

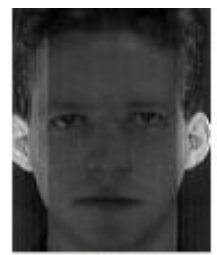

(a)

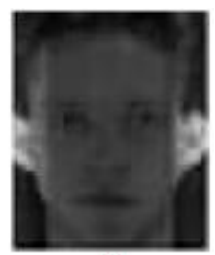

(b)

Figure 3. 50-dimensional reconstruction of the map contrast before and after the compression

In order to measure the effectiveness of this method, the concept of compression ratio is proposed and compared with the pure NMF method and the simple DCT method respectively. It is necessary to explain here that the NMF method uses the original image bit number and reduces to $50, \mathrm{DCT}$ is the ratio of the original image to the number of compressed image bits, The NMF-DCT method is the ratio of the number of bits in the original image after the dimension reduction. The results are shown in the following table:

Table 2 Compression ratios of the three methods

\begin{tabular}{cccc}
\hline Methods & NMF & DCT & NMF-DCT \\
$C_{r}$ & 1.452 & 2.776 & 4.560 \\
\hline
\end{tabular}

It can be concluded from Table 2 that the compression ratio of the three methods tends to increase, because the proposed method can effectively reduce the redundancy in the image. The information entropy redundancy can be eliminated to improve the compression ratio of the image. 
The superiority of this method in dimension reduction of face images is obvious. This experiment also has some shortcomings, the reconstructed image matrix $V^{\prime}$ is extended to increase the data volume, which is convenient to deal with but increase the burden of system processing. This part of the content remains to be optimized.

\section{Conclusion}

NMF-DCT method is a dimension reduction method which combines the advantages of the two methods. The base matrix $W$ and the projection coefficient matrix myH are obtained by non-negative matrix decomposition of the original matrix $V$, and the compressed data is obtained by compressing the reconstructed matrix $V^{\prime}$. In this paper, a face in the ORL face database is used to reduce the dimension of the face. According to the fit degree formula, the best reconstruction effect is obtained when the dimension $r=50$. Compared with the NMF method and the DCT method, the NMF-DCT method has better compression effect. Experimental results show that the NMF-DCT method has a good effect on image dimension reduction. It will facilitate the transmission for images.

\section{Acknowledgment}

This work is supported by National Natural Science Foundation of China under Grant No.51105291.

\section{References}

[1] L.J.P. Maaten, E.O. Postma and H.J. Herik. Dimensionality Reduction: a Comparative Review [J]. IEEE Trans. On Pattern Analysis and Machine Intelligence, Vol. 10 (2007) No.2, p.1.

[2] D.D. Lee, H.S. Seung. Learning the Parts of Objects with Non-negative Matrix Factorization, Nature, Vol. 401 (1999) p.788.

[3] X.T. Wu, D.L. Yan. Analysis and Research on Data Dimension Reduction Method [J]. Application Research of Computers, Vol. 26 (2009) No.8, p.2833. (In Chinese)

[4] Z. Zhang, H.X. Ni and C.M. Yuan. Digital Image Processing and Recognition [M]. (People's Posts and Telecommunications Press, China 2013). (In Chinese)

[5] L. Li, Y.J. Zhang. Review on Non-negative Matrix Factorization Algorithm [J]. Journal of Electronics, Vol. 36 (2008) No.4, p.738. (In Chinese)

[6] Y.J. Zhang, Z.D. Cheng. Face Recognition Based on Subspace [M]. (Tsinghua University Press, China 2009), p.78. (In Chinese)

[7] Z.H. Gao, W.B. Xu. MATLAB-based Image Processing Case Tutorial [M]. Beijing: Tsinghua University Press, China 2011). (In Chinese)

[8] Z.W. Zhang, F. Yang. Face Recognition Method Based on Wavelet Transform and the NMF Research [J]. Computer engineering, Vol. 33 (2007) No.6, p.176. (In Chinese)

[9] Y. Zhang. MATLAB Image Processing Super Learning Manual [M]. (People's Posts and Telecommunications Press, China 2014). (In Chinese)

[10] L.J. Kong. MATLAB Wavelet Analysis of the Super Learning Manual [M]. (People's Posts and Telecommunications Press, China 2014). (In Chinese) 